\title{
Lectin Binding to Neurites of Goldfish Retinal Explants
}

\author{
EVA L. FELDMAN, ANNE M. HEACOCK and B. W. AGRANOFF* \\ The University of Michigan, Neuroscience Laboratory, Ann Arbor, MI 48109 (U.S.A.)
}

(Accepted March 4th, 1982)

\begin{abstract}
Key words: concanavalin A — fluorescence microscopy — goldfish — lectin binding — retinal explants - ricin wheat germ agglutinin
\end{abstract}

\begin{abstract}
The lectin binding characteristics of goldfish retinal explants were examined by fluorescence microscopy. Neurites grown out from cultured retinal explants were found to bind concanavalin A, wheat germ agglutinin and ricin (agglutinin). The effects of tissue fixation on lectin binding to retinal explant neurites suggest that glycolipids may constitute the predominant ricin binding sites. A reduction in labeling with wheat germ agglutinin following sialidase treatment indicates preferential binding of the lectin to sialic acid residues in the neurite membrane. Neurite morphology was unaltered by brief exposure to concanavalin $\mathrm{A}$ or wheat germ agglutinin, while ricin caused a marked deterioration.
\end{abstract}

\section{INTRODUCTION}

We have previously demonstrated that retinal explants exhibit vigorous neuritic outgrowth, which has been shown to derive from ganglion cells ${ }^{11}$, providing the optic nerve had been crushed 10-15 days previously ${ }^{12}$. They thus provide an in vitro preparation for studies of the regenerating axon in the absence of supporting cells. In this report, we have employed fluorescent plant lectins to examine the nature of the carbohydrate constituents of axonal membrane glycoconjugates.

\section{MATERIALS AND METHODS}

Common goldfish, Carassius auratus (7-11 g), were purchased from Ozark Fisheries (Stoutland, $\mathrm{MO}$ ) and maintained in constant light at $21 \pm 1{ }^{\circ} \mathrm{C}$ in aerated aquaria. Right optic nerve crush was performed as described previously ${ }^{12}$.

Rhodamine-conjugated Ricinus communis agglutinin (Rh-RCA I, MW 120,000) wheat germ agglutinin lectin ( $R h-W G A)$, concanavalin A ( $R h-C o n$ A), the biotinylated lectins: Dolichos biflorus lectin (b-DBA), Ulex europeus I lectin (b-UEA), peanut agglutinin (b-PNA) and rhodamine-conjugated avidin were purchased from Vector Laboratories. Fluorescein-conjugated DBA (FITC-DBA) and fluorescein-conjugated UEA (FITC-UEA) were products of E-Y Lectins. Fluorescein-conjugated Bandeiraea simplicifolia $\mathbf{B}_{4}$ isolectin (FITC-BS$B_{4}$ ), and $\mathrm{N}, \mathrm{N}^{\prime}, \mathrm{N}^{\prime \prime}$-triacetylchitotriose, were generous gifts of Dr. Irwin Goldstein. Concentrated stock solutions of lectins were stored at $-20{ }^{\circ} \mathrm{C}$. Prior to use, the solution was thawed, diluted in phosphate buffered salts (PBS) ${ }^{4}$ and assayed for activity by the capillary tube test ${ }^{19}$. All other materials used in these studies were obtained from standard commercial sources.

\section{Lectin binding to retinal explant cultures}

Ten to 14 days after optic nerve crush, retinas were explanted onto polylysine coated coverslips or plastic dishes and grown in supplemented L-15 (Leibowitz) tissue culture medium as previously described ${ }^{10,12}$.

In the following experiments, unless otherwise indicated, all cultures were rinsed 3 times $(10 \mathrm{~min}$ per rinse) in PBS, and all incubations were in PBS at room temperature, in a volume sufficient to barely cover the explants. Four- to 7-day-old cultures were rinsed and incubated with $25-200 \mu \mathrm{g} / \mathrm{ml}$ lectin or

\footnotetext{
* To whom all correspondence should be addressed.
} 

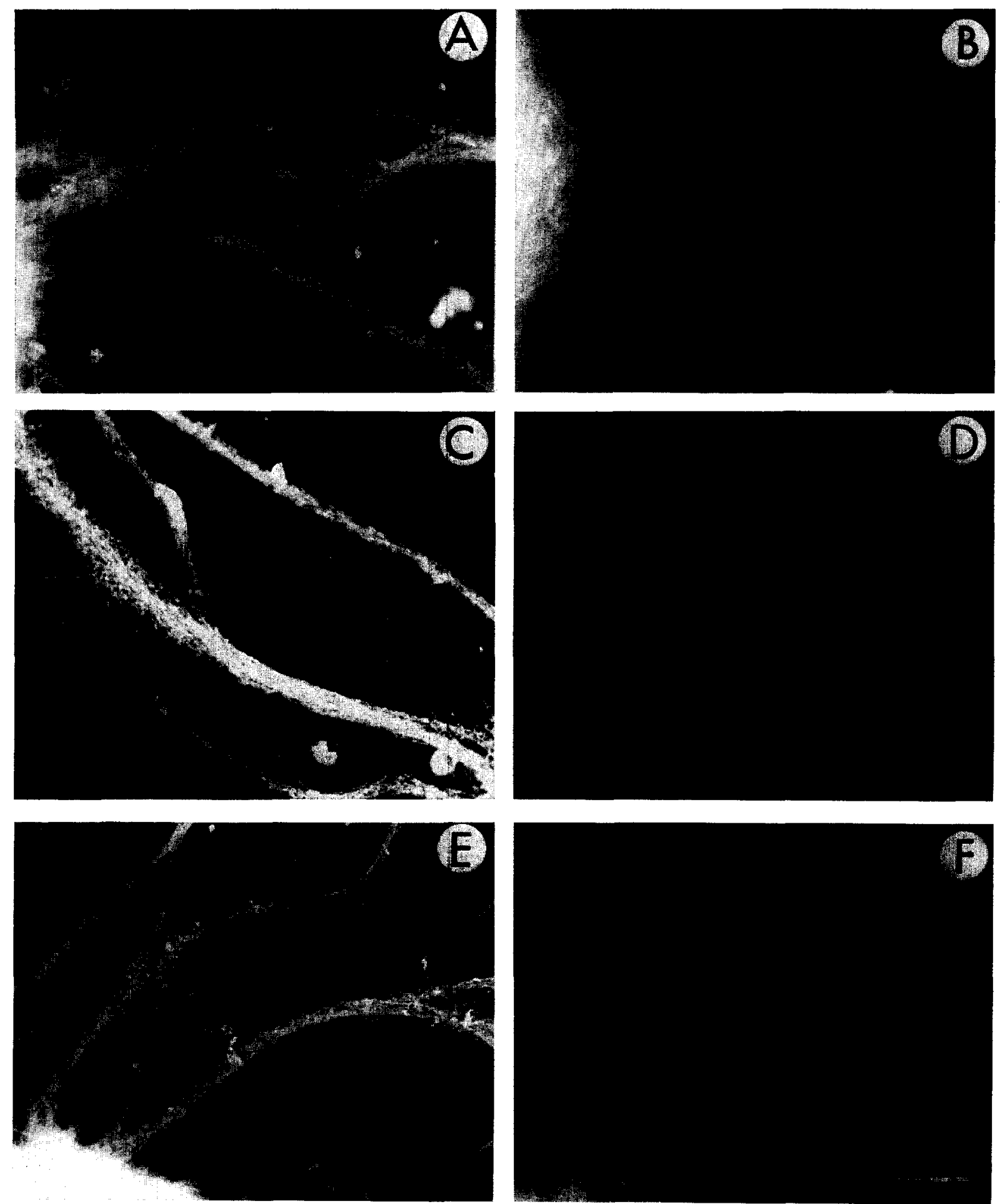

Fig. 1. Lectin binding to explant cultures of adult goldfish retina. Fluorescent photomicrographs of retinal explant cultures labeled with $200 \mu \mathrm{g} / \mathrm{ml} \mathrm{Rh-Con} \mathrm{A} \mathrm{(A),} \mathrm{Rh-WGA} \mathrm{(C)} \mathrm{and} \mathrm{Rh-RCA} \mathrm{I} \mathrm{(E)} \mathrm{and} \mathrm{their} \mathrm{respective} \mathrm{competing} \mathrm{sugar} \mathrm{controls} \mathrm{(B,} \mathrm{D} \mathrm{and} \mathrm{F).} \mathrm{Bar}$ $=50 \mu \mathrm{m}$. 
lectin plus sugar control solution for $30 \mathrm{~min}$, rinsed again and then observed with fluorescence microscopy. In a second and more sensitive procedure, 6day-old cultures were incubated with either b-DBA, b-UEA or b-PNA for $45 \mathrm{~min}$, rinsed, then incubated with rhodamine-conjugated avidin (200 $\mu \mathrm{g} / \mathrm{ml}$ ) for $45 \mathrm{~min}$.

The following sugars $(0.1 \mathrm{M})$ were used as controls for non-specific binding: $\alpha$-methylmannoside (Con A), triacetylchitotriose (20 mM, WGA), lactose (RCA I; PNA), N-acetyl-D-galactosamine (DBA), fucose (UEA) and methyl $\alpha$-D-galactose (BS-B ${ }_{4}$ ).

For examination of the effect of prior fixation, 4to 7-day cultures were rinsed, then fixed either in AFA ( $80 \%$ aqueous ethanol:formalin:glacial acetic acid, $90: 5: 5, \mathrm{v} / \mathrm{v} / \mathrm{v}$ ) for $1 \mathrm{~h}$ or in chloroform:metha- nol $(2: 1)$ overnight. Following fixation, the cultures were rinsed, then incubated with the appropriate lectin solution. For double label experiments, cultures were first tagged with FITC-Con A or FITCWGA then incubated with a fluorescent lipid dye, dioctadecylindocarbocyanine (dil), a gift of Dr. Daniel Axelrod, at a concentration of $2.5 \mu \mathrm{g} / \mathrm{ml}$ in PBS with $1 \%$ ethanol for $10 \mathrm{~min}$ at $37^{\circ} \mathrm{C}$. At the end of the incubation, cultures were rinsed and examined for both fluorescein (Leitz Filter System I) and diI fluorescence (Leitz Filter System M with 480 $\mathrm{nm}$ edge filter).

In other experiments, cultures were pre-treated with 0.2 units $/ \mathrm{ml}$ of sialidase (Sigma, Type IX neuraminidase) in isotonic citrate-phosphate buffer, $\mathrm{pH} \mathrm{6.2,} \mathrm{for} 1 \mathrm{~h}$ prior to lectin incubations.

For determination of the short-term toxic effects
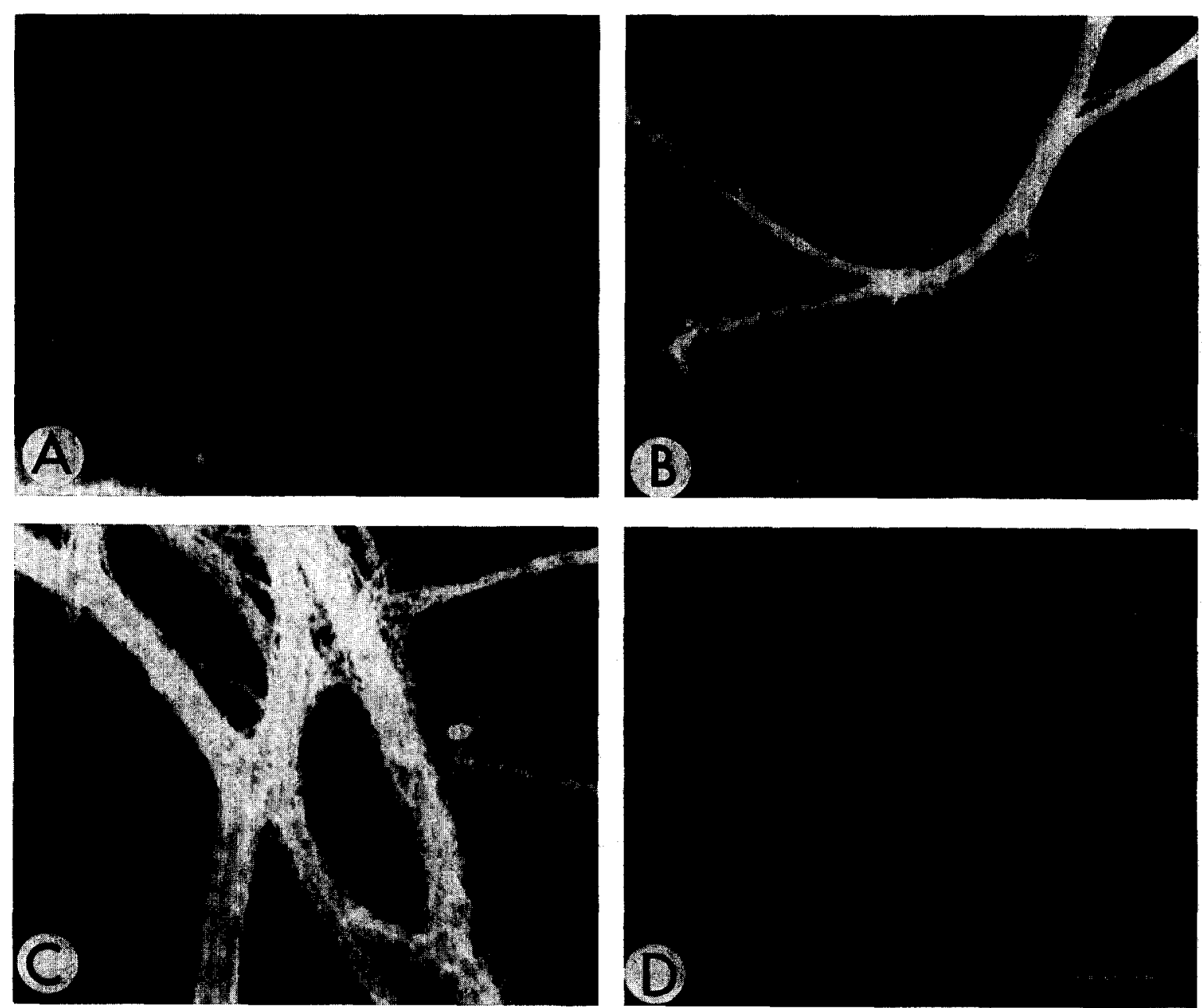

Fig. 2. The effect of neuraminidase treatment on $R h-R C A$ I and $R h-W G A$ binding to retinal explants. Control (A, C) or neuraminidase-treated (B, D) explants labeled with $100 \mu \mathrm{g} / \mathrm{ml} \mathrm{Rh}-\mathrm{RCA}$ I $(\mathrm{A}, \mathrm{B})$ or Rh-WGA $(\mathrm{C}, \mathrm{D})$. Bar $=50 \mu \mathrm{m}$. 

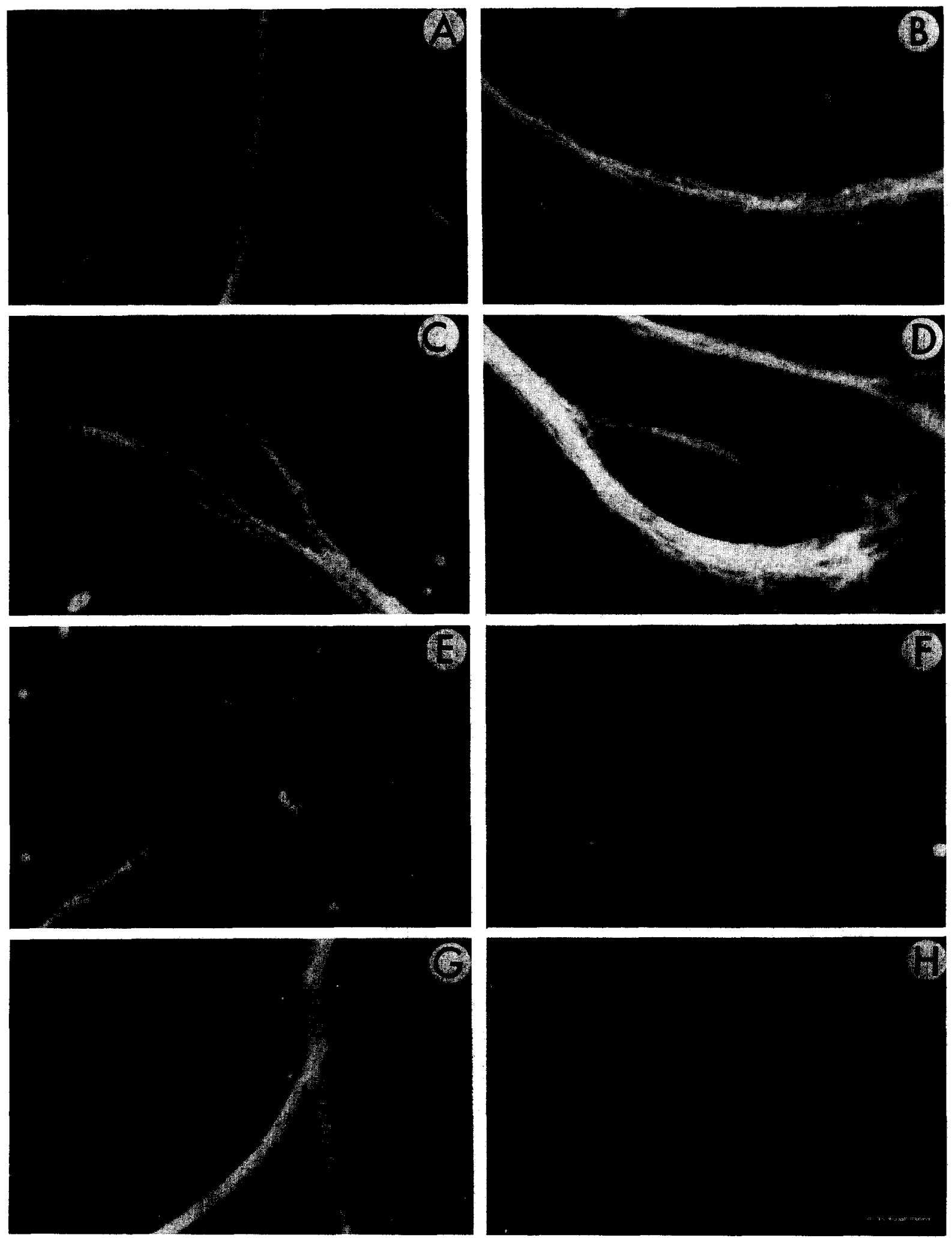

Fig. 3. The effect of fixation on lectin binding to retinal explants. Unfixed cultures (A, C and E) or chloroform:methanol fixed cultures (B, D and F) labeled with $200 \mu \mathrm{g} / \mathrm{ml} \mathrm{Rh-Con} \mathrm{A} \mathrm{(A} \mathrm{and} \mathrm{B),} \mathrm{Rh-WGA} \mathrm{(C} \mathrm{and} \mathrm{D)} \mathrm{or} \mathrm{Rh-RCA} \mathrm{I} \mathrm{(E} \mathrm{and} \mathrm{F).} \mathrm{FA-treated} \mathrm{(G)}$ and AFA-treated $(\mathrm{H})$ cultures labeled with $200 \mu \mathrm{g} / \mathrm{ml}$ Rh-RCA I. Bar $=50 \mu \mathrm{m}$. 
of RCA I, cultures, at day 6 in vitro, were rinsed twice with PBS, incubated with $0.05-5 \mu \mathrm{g} / \mathrm{ml} \mathrm{RCA} \mathrm{I}$ in PBS for $30 \mathrm{~min}, 1 \mathrm{~h}$ or $3 \mathrm{~h}$, rinsed again, and then assessed photomicrographically.

\section{RESULTS}

\section{Lectin binding to retinal explant neurites}

Fluorescence microscopy following incubation of the cultures with Rh-Con A, Rh-WGA and RhRCA I revealed binding to the neurites (Fig. 1A, C and $E$ ), which was absent in the respective control incubations with competing sugars (Fig. 1B, D and F). Even with the more sensitive rhodamine avidinbiotinylated lectin assay, UEA, DBA and PNA (200 $\mu \mathrm{g} / \mathrm{ml})$ showed no binding, nor did FITC-BS-B 4 lectin, which was not available in biotinylated form. That the observed retinal explant lectin binding sites are most likely confined to the surface of the neurite membrane is indicated by previous studies in which antibody to Con $\mathrm{A}$ was found to bind to Con A labeled neurites ${ }^{5}$ and by electron microscopic examination of binding of ferritin-tagged lectins (unpublished observations).

Rh-RCA I binding could be substantially increased by pretreatment of the cultures with sialidase (Fig. 2A, B) which presumably exposes galactose residues masked by sialic acid. $\mathrm{Rh}-$ Con A labeling was unaffected by this treatment (not shown) while Rh-WGA binding was markedly reduced following removal of sialic acid (Fig. 2C, D).

\section{Effect of fixation}

Treatment with chloroform:methanol $(2: 1)$ had no effect on the binding of Rh-Con A (Fig. 3A, B) or Rh-WGA to the neurites (Fig. 3C, D), but caused a substantial reduction of Rh-RCA I binding (Fig. $3 E, F)$. RCA $I$ is known to bind to glycolipids as well as glycoproteins ${ }^{8}$. Since the fixative used here would be expected to extract glycolipids from the neurite membrane, the decrease in RCA I binding suggests that glycolipid is the major RCA I binding site. Alternatively, RCA I binding to glycoprotein may be particularly sensitive to fixation, even in the absence of organic solvents. We therefore compared the effect of AFA fixation to that of treatment with 'FA' (the same mixture with water replacing the alcohol). The reduced RCA I labeling seen with AFA was not observed with FA (Fig. 3G, H). These results are consistent with the glycolipid nature of the RCA I binding sites in retinal explant neurites.

\section{Lectin binding to growth cones}

With all three lectins, at all concentrations examined, there was generally an apparent increased fluorescence at the growth cone region. To determine whether there was indeed an enrichment of lectin receptors at the growth cone or simply enhanced labeling due to the presence of more surface membrane at the growing tip, we compared the binding pattern of fluorescent lectins with that of a lipophilic dye, diI. This fluorescent probe intercalates with existing lipid bilayers and provides a highly resolved outline of membranous structures ${ }^{1}$. Examination of diI-labeled cultures revealed much detailed structure including numerous microspikes, filopodia and flattened extensions, not previously apparent. Double label experiments revealed that the increased lectin fluorescence at the growth cone corresponded with increased diI fluorescence (Fig. 4). The enhanced fluorescence seen at the growth cone appears then to be due to an increase in the available membrane rather than to enrichment in lectin binding sites. In a recent quantitative study, Pfenninger has reported some differences in the density of lectin receptors on neurites and growth cones $^{17}$.

\section{Effects of lectins on neurite morphology}

Neurite morphology, as assessed by phase microscopy, was unaltered by brief exposure to Con $\mathrm{A}$ or WGA (at $1-200 \mu \mathrm{g} / \mathrm{ml}$ ). However, within $30 \mathrm{~min}$ following RCA I treatment $(5 \mu \mathrm{g} / \mathrm{ml})$ the neurites exhibited an altered appearance, notably a flattening of the growth cone and filopodia, with obvious deterioration occurring by $3 \mathrm{~h}$ (Fig. 5). Removal of the RCA I following the brief exposure did not prevent the eventual complete degeneration of the neurites although their deterioration was substantially delayed (by up to $24 \mathrm{~h}$ ) if the RCA I was added together with its competing sugar, lactose. Decreasing the concentration of RCA I to 0.5 or $0.05 \mu \mathrm{g} / \mathrm{ml}$ also resulted in a correspondingly delayed onset of degeneration, though even the lowest dose examined elicited deterioration by $24 \mathrm{~h}$. 

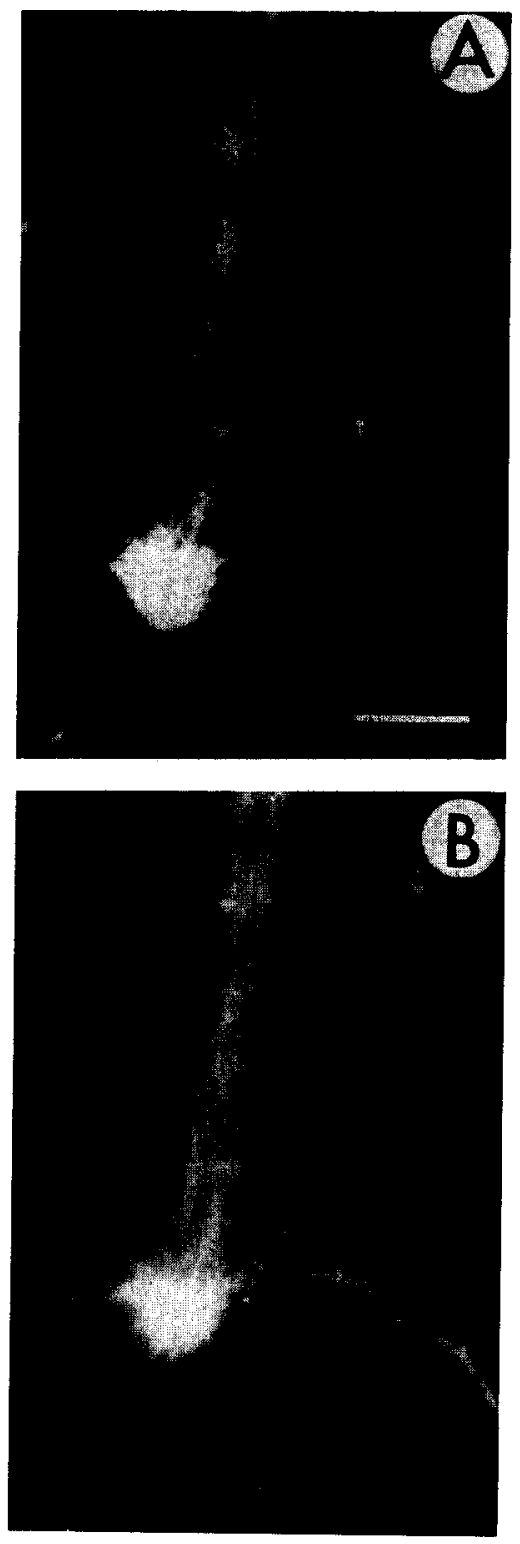

Fig. 4. Neurites labeled with Con A and the lipophilic dye, diI. Doubly labeled culture photographed with fluorescein filters to show FITC Con A labeling (A) and the same field photographed with rhodamine filters to show diI labeling (B). Bar $=15 \mu \mathrm{m}$.

\section{DISCUSSION}

The carbohydrate nature of regenerating optic nerve was investigated by examining the lectin binding properties of goldfish retinal explant neurites in vitro. Lectins specific for $\alpha$-methylmannoside, $\mathrm{N}$ acetyl-glucosamine and $\beta$-galactose were found to bind to the neurites while those with affinity for fucose, $\mathrm{N}$-acetyl-galactosamine and $\alpha$-galactose did not bind. This lack of binding indicates that the latter sugar residues are either inaccessible or present in amounts too low to be detectable, even by the sensitive biotin-avidin method. Binding of Con A to chick retina ${ }^{13}$ and neuroblastoma cultures ${ }^{6}$ has been reported while fetal rat and chick superior cervical ganglion, spinal cord and cerebellar cultures bind Con A, WGA and RCA $1^{2,15}$. DBA has only been found to bind to rat superior cervical ganglion culture while no binding sites for UEA have been detected $^{16}$.

Determination of the effects of fixation or of enzyme treatment has given further information as to the nature of the lectin binding sites on the ganglion cell axons. A fixation mixture containing an organic solvent caused a decrease in RCA I binding to the optic tectum as well as the retinal explant neurites, but had no effect on the binding of Con A or WGA. These observations suggest two possible explanations: (1) glycoprotein binding sites for RCA I, which remain intact following aqueous fixation (FA), are destroyed or become inaccessible following treatment with organic solvents; or (2) RCA I binding sites are predominantly extractable glycolipids while Con A and WGA bind mainly to glycoproteins. Since RCA I binding to glycolipids has been described ${ }^{8}$, the latter explanation seems the most likely. Our results are similar to those of Pfenninger and Maylie-Pfenninger ${ }^{16}$ who found that chloroform-methanol extraction of rat sympathetic ganglia cultures decreased RCA I binding but had no effect on WGA binding. It should be noted, however, that Fuchtbauer and Schachner ${ }^{7}$ have reported effects of fixation on Con A binding to mouse cerebellar sections.

The sialosyl $(\alpha 2 \rightarrow 3)$ galactosyl sequence is common to both glycolipid and glycoprotein oligosacchari$\mathrm{des}^{8}$. Sialidase treatment of the retinal explants enhanced RCA I binding by exposing galactosyl residues while WGA binding was decreased following removal of the terminal sialic acid residues. WGA is known to bind sialic acid as well as Nacetyl-glucosamine ${ }^{8}$ and pretreatment of synaptic membranes with sialidase has been reported to decrease WGA binding by $80 \%$. In light of the effects of fixation and sialidase treatment on lectin binding, these data suggest that RCA I is binding to 

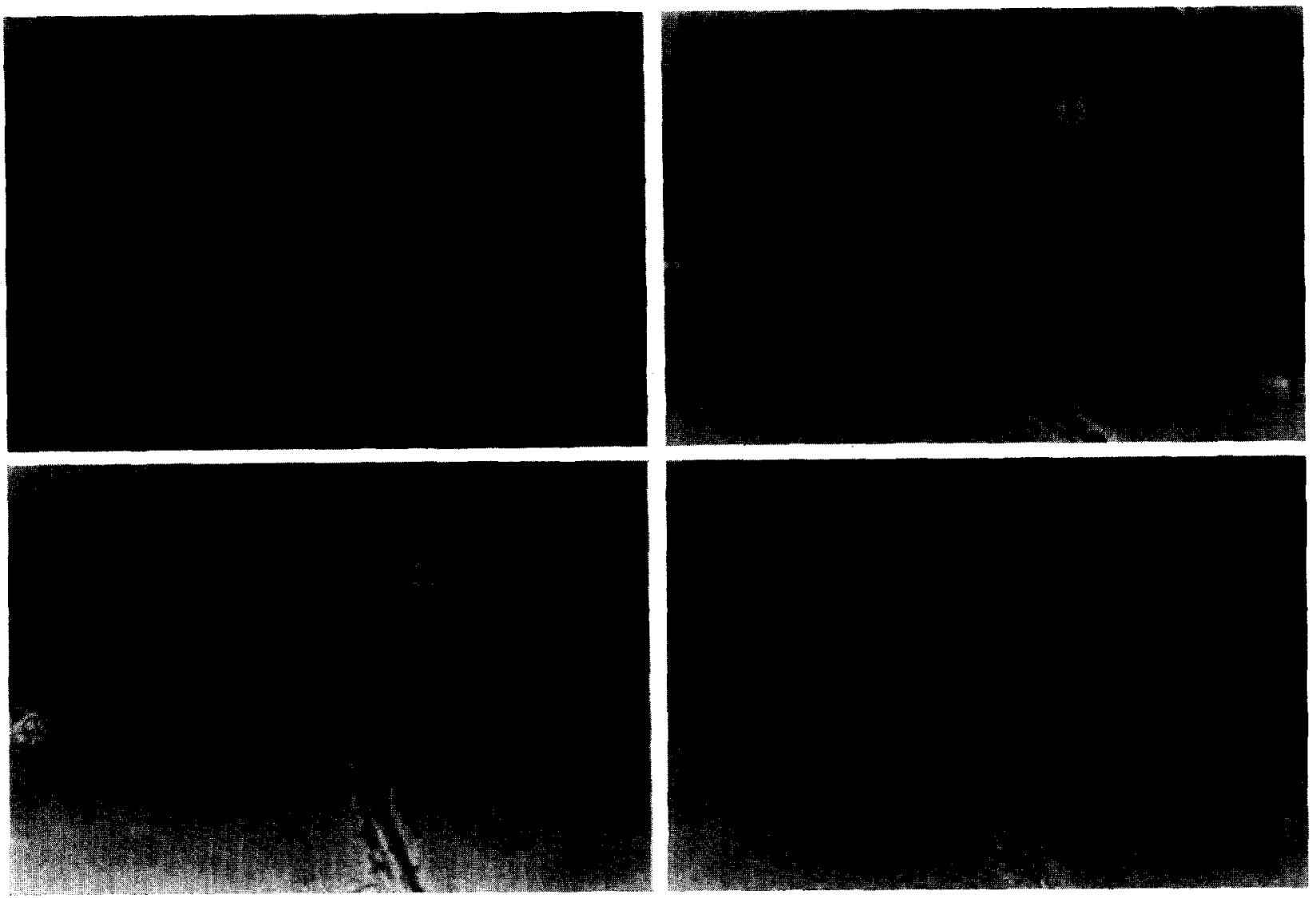

Fig. 5. RCA I effects on neurite morphology. Sequential photomicrographs of a culture before (A) and after $30 \mathrm{~min}$ (B), $1 \mathrm{~h}(\mathrm{C})$ and $3 \mathrm{~h}$ (D) exposure to $5 \mu \mathrm{g} / \mathrm{ml} \mathrm{RCA} \mathrm{I}$ in PBS. Bar $=50 \mu \mathrm{m}$.

terminal galactosyl residues of glycolipids, while glycoproteins with terminal sialic acid residues make a substantial contribution to WGA binding. These observations are in agreement with a recent study by McLaughlin et al. ${ }^{14}$ reporting a decrease in WGA binding and an increase in RCA I binding after sialidase treatment of chick retina.

RCA I binding produced marked changes in neurite morphology (Fig. 5). While RCA $I$ is not nearly as toxic as RCA II, its $\alpha$ subunits can inhibit protein synthesis ${ }^{8}$. It seems, however, unlikely that the structural changes seen in the neurites after RCA I treatment reflected a block of protein synthesis, since the changes occurred rapidly. Indeed, no alterations in neurite morphology are observed for

\section{REFERENCES}

1 Axelrod, D., Wight, A., Webb, W. and Horwitz, A., Influence of membrane lipids on acetylcholine receptor and lipid probe diffusion in cultured myotube membrane, several days after treatment of the explants with cycloheximide $^{9}$. It appears that some other toxic mechanisms must be at work. There is data to suggest that RCA I may be more toxic to nervous than to non-nervous tissue ${ }^{18}$, and it is possible that the observed alterations in neurite morphology after RCA I addition may be related to the lectin's neurotoxicity.

\section{ACKNOWLEDGEMENTS}

The generous gifts of Dr. Irwin Goldstein and Dr. Daniel Axelrod of The University of Michigan are gratefully acknowledged. This research was supported by Grant NS 13743.

Biochemistry, 17 (1978) 3604-3609.

2 Carbonetto, S. and Argon, Y., Lectins induce the redistribution and internalization of receptors on the surface of cultured neurons, Develop. Biol., 80 (1980) 364-378.

3 Cruz, T. F. and Gurd, J. W., Reaction of synaptic plasma 
membrane sialoglycoproteins with intrinsic sialidase and wheat germ agglutinin, J. biol. Chem., 253 (1978) 7314-7318.

4 Dulbecco, R., Plaque formation and isolation of pure lines with poliomyelitis viruses, $J$. exp. Med., 99 (1954) 167-199.

5 Feldman, E. L., Axelrod, D., Schwartz, M., Heacock, A. M. and Agranoff, B. W., Studies on the localization of newly added membrane in growing neurites, $J$. Neurobiol., 12 (1981) 591-598.

6 Fishman, M. C., Dragsten, P. R. and Spector, I., Immobilization of concanavalin A receptors during differentiation of neuroblastoma cells, Nature, 290 (1981) 781-783.

7 Fuchtbauer, A. and Schachner, M., Effects of aldehydes and organic solvents on concanavalin A binding sites in cerebellar tissue sections, Neurosci. Lett., 12 (1979) 355-360.

8 Goldstein, I. J. and Hayes, C., The lectins: carbohydratebinding proteins of plants and animals, Advanc. Carb. Chem. Biochem., 35 (1978) 127-340.

9 Heacock, A. M., Biosynthetic requirements for neurite outgrowth in goldfish retina explants, Neurosci. Abstr., 5 (1979) 756.

10 Heacock, A. M. and Agranoff, B. W., Clockwise growth of neurites from retinal explants, Science, 198 (1977) 64-66.

11 Johns, P. R., Heacock, A. M. and Agranoff, B. W., Neurites in explant cultures of adult goldfish retina derive from ganglion cells, Brain Research, 142 (1977) 531-537.
12 Landreth, G. E. and Agranoff, B. W., Explant culture of adult goldfish retina: a model for the study of CNS regeneration, Brain Research, 161 (1979) 39-53.

13 Letourneau, P. C., Inhibition of intracellular adhesion by concanavalin $\mathrm{A}$ is associated with concanavalin A-mediated redistribution of surface receptors, J. Cell Biol., 80 (1979) 128-140.

14 McLaughlin, B. J., Wood, J. G. and Gurd, J. W., The localization of lectin binding sites during photoreceptor synaptogenesis in the chick retina, Brain Research, 191 (1980) 345-357.

15 Pfenninger, K. H. and Maylie-Pfenninger, M. F., Characterization, distribution and appearance of surface carbohydrates on growing neurites. In A. Karlin, V. Tennyson and H. Vogel (Eds.), Neuronal Information Transfer, Academic Press, New York, 1978, pp. 373-386.

16 Pfenninger, K. H. and Maylie-Pfenninger, M. F., Surface glycoconjugates in the differentiating neuron. In $\mathrm{R}$. V. Margolis and R. K. Margolis (Eds.), Complex Carbohydrates of Nervous Tissue, Plenum Press, New York, 1979, pp. 185-191.

17 Pfenninger, K. H. and Maylie-Pfenninger, M. F., Lectin labeling of sprouting neurons. I. Regional distribution of surface glycoconjugates, $J$. Cell Biol., 89 (1981) 536-546.

18 Strocchi, P., Novello, F., Montavaro, N. and Stirpe, F., Effect of intraventricularly injected ricin on protein synthesis in rat brain, Neurochem. Res., 4 (1979) 259-263.

19 Swift, H., Wilson, A. T. and Lancefield, R. C., Typing group $\mathbf{A}$ hemolytic streptococci by $\mathbf{M}$ precipitin reactions in capillary pipettes, J. exp. Med., 78 (1943) 127-133. 